\title{
Progenitor's Signatures in Type Ia Supernova Remnants
}

\author{
A. Chiotellis ${ }^{1}$, D. Kosenko ${ }^{1}$, K. M. Schure ${ }^{1,2}$, and J. Vink ${ }^{1}$ \\ ${ }^{1}$ Sterrenkundig Instituut Utrecht, \\ Postbus 80000, NL-3508TA, Utrecht, the Netherlands \\ email: a.chiotellis@astro-uu.nl \\ ${ }^{2}$ Department of Physics, University of Oxford, \\ Clarendon Laboratory, Parks Road, Oxford OX1 3PU, United Kingdom
}

\begin{abstract}
The remnants of Type Ia supernovae (SNe Ia) can provide important clues about their progenitor histories. We discuss two well-observed supernova remnants (SNRs) that are believed to have resulted from SNe Ia, and use various tools to shed light on the possible progenitor histories. We find that Kepler's SNR is consistent with a symbiotic binary progenitor consisting of a white dwarf and an AGB star. Our hydrosimulations can reproduce the observed kinematic and morphological properties. For Tycho's remnant we use the characteristics of the X-ray spectrum and kinematics to show that the ejecta has likely interacted with dense circumstellar gas.
\end{abstract}

Keywords. supernova remnants - supernovae: individual (SN 1604, SN 1572) - binaries: symbiotic

\section{Introduction}

Type Ia supernovae (SNe Ia) have been the key to the discovery that the universe is accelerating. In addition, they comprise one of the main sources of the chemical enrichment in galaxies with iron peak elements. Given their importance, it is disconcerting that their nature is still poorly understood. SNe Ia are believed to result from the thermonuclear explosion of a $\mathrm{CO}$ white dwarf (CO WD) which approaches the Chandrasekhar mass through mass accretion from a companion star. However, the nature of the donor star, the binary evolution path that leads to SNe Ia, and the explosion mechanism are still unclear. Different evolutionary paths of Type Ia progenitors lead to different modifications of the ambient medium, either through mass outflow, or through ionizing radiation that can accompany accretion. The subsequent interaction of the supernova ejecta with the modified (or not) circumstellar medium (CSM) leads to different properties of the SNRs (morphology, dynamics, spectra etc.). Thus, the local population of Type Ia SNRs can provide us with valuable information about Type Ia progenitors.

Here we model two historical SNe: SN1604 (Section 2) and SN1572 (Section 3). In both cases we study the impact of the interaction between the SN ejecta and dense circumstellar structures on the observational properties of these SNRs.

\section{The Case of Kepler's SNR (SN 1604)}

Kepler's SN occurred in 1604 high above the Galactic plane (G4.5+6.8). Its radius is $2.6 d_{5} \mathrm{pc}$, with $d_{5}$ the distance in units of $5 \mathrm{kpc}$. This SNR has been a puzzling object due to its increased emissivity in the northern region, which shows a substantial overabundance of nitrogen, $\mathrm{N} / \mathrm{N}_{\odot}>2$, but otherwise solar metallicity (Blair et al. 1991). 
The presence of the nitrogen-rich shell appears to affect the dynamics. In the north, the expansion parameter $[m=(d R / d t) /(R / t)=0.35]$ is lower than the rest of the SNR $[\mathrm{m}=0.6$ (Vink 2008)], and lower than expected for young SNRs $(m>0.4)$. All of the aforementioned properties indicate the existence of a massive shell in the northern region that was formed by mass outflows during the evolution of the progenitor system.

We have shown that these characteristics can be explained if the CSM was shaped by the stellar wind of an asymptotic giant branch (AGB) donor star (Chiotellis et al. 2011). AGB stars with initial masses $>4 \mathrm{M}_{\odot}$ are able to enrich their surfaces with nitrogen. Based on the AGB models of Karakas \& Lattanzio (2007), the chemical composition of the circumstellar shell at Kepler's SNR can be best explained if the AGB donor star had an initial mass of $(4-5) \mathrm{M}_{\odot}$ and solar metallicity. This suggests that the progenitor system was a wide symbiotic binary, where part of the slow wind of the donor has been accreted onto the WD while the rest of the wind formed the observed nitrogen-rich shell. Finally, we retain the idea, first suggested by Bandiera (1987), that the asymmetry of the northern shell of Kepler's SNR can be explained by its observed supersonic motion of $250 \mathrm{~km} \mathrm{~s}^{-1}$ away from the Galactic plane (Bandiera \& van den Bergh 1991; Sollerman et al. 2003). The interaction of the stellar wind with the ram pressure of the ISM leads to the formation of a bow-shaped shell. Nowadays, the SNR's blast wave interacts only with the nearest region of this bow shell.

In order to test this scenario we have performed 2D hydrosimulations employing the AMRVAC code (Keppens et al. 2003). First we simulate the formation of the CSM by imposing an inflow with the properties of a spherical, cold and slow stellar wind. At the same time the ISM with constant density $\left(\rho_{i s m}\right)$ enters from one side with momentum $m=\rho_{i s m} u_{*}$, to represent the systemic motion of the progenitor system with velocity $u_{*}$ (see Figure 1) and forms the bow-shaped shell. In the second stage, we introduce the supernova ejecta and let the SNR evolve. Figure 1 shows the result of the simulation at the current age of Kepler's SNR. The model reproduces the observed characteristics of Kepler's SNR: the remnant interacts with the nearest region of the bow shell only, explaining the observed asymmetry, and has an expansion rate inside the shell of $m=$ $0.3-0.35$ versus $m=0.6$ in the rest of the remnant, in agreement with the observations.
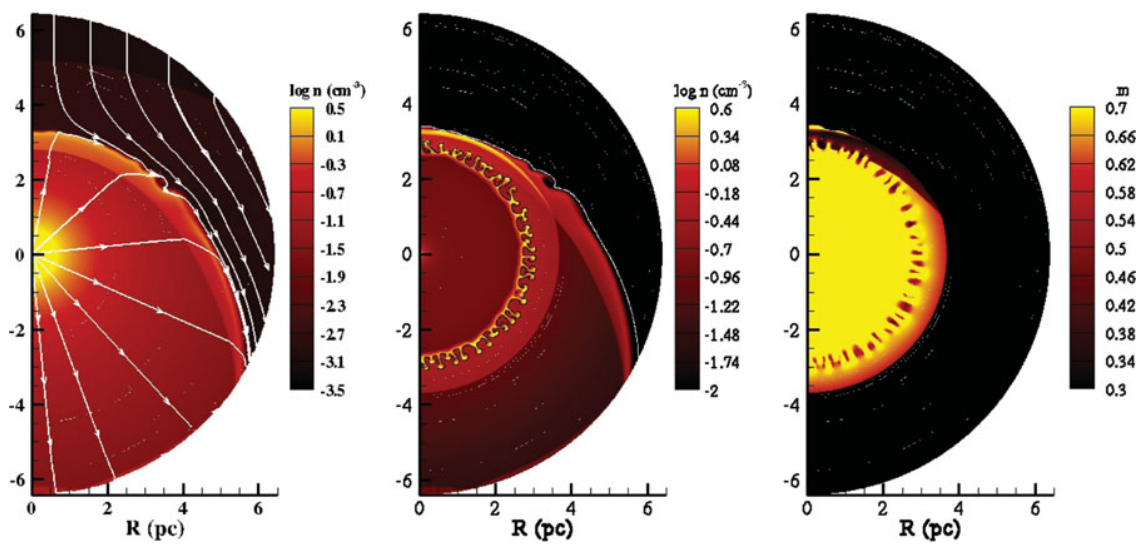

Figure 1. Left: The formation of the bow-shaped shell. The wind parameters that have been used are: mass loss rate $\dot{M}=10^{-5} \mathrm{M}_{\odot} \mathrm{yr}^{-1}$, wind velocity: $u_{w}=10 \mathrm{~km} \mathrm{~s}^{-1}$, ISM density $n_{\text {ism }}=7 \times 10^{-4} \mathrm{~cm}^{-3}$, systemic velocity $u_{*}=250 \mathrm{~km} \mathrm{~s}^{-1}$ while the timescale of the bubble evolution is $0.38 \mathrm{Myr}$. The density and the expansion parameter of the SNR at the current age of Kepler's SNR are depicted in the middle and right panel respectively. The energy of the SN is $10^{51} \mathrm{erg}$ while the mass of the ejecta is $1.4 \mathrm{M}_{\odot}$ (Chiotellis et al. 2011). 


\section{The Case of Tycho's SNR (SN 1572)}

The case for a CSM shaped by the progenitor for Kepler is quite obvious. But what about other Type Ia SNRs? For another important historical SNR, SN 1572 /Tycho's SNR, the case for a CSM shell is less conspicuous. However, there is a clear discrepancy between the ISM densities as measured by modeling the X-ray emission from a delayeddetonation explosion (Badenes et al. 2006), $2 \times 10^{-24} \mathrm{~g} \mathrm{~cm}^{-3}$, and density estimates based on the kinematics of the SNR. The latter indicating a density that is five times lower (Katsuda et al. 2010). For this reason Katsuda et al. (2010) have suggested that a more complex circumstellar structure may resolve this discrepancy.

To study the effects of a dense shell, we have simulated the X-ray emission from two SNRs; one evolving into a homogeneous ISM, and the other interacting with a CSM that was formed by a stellar wind, before propagating further into a homogeneous ISM.

We performed our simulations in three steps. Initially, using the AMRVAC code, we form the ambient medium for the wind profile (first column of Figure 2). Subsequently, we let the SNR evolve in either a homogeneous ISM, or in the CSM+ISM, using the hydrodynamical code SUPREMA (Sorokina et al.2004, Kosenko et al. 2011) with the W7 deflagration explosion model (Nomoto 1984). Finally, we calculate the X-ray emission from the simulated SNRs employing the SPEX software package (Kaastra et al. 1996).

We produced a number of simulations with various wind parameters. Figure 2 shows a typical example that fits the characteristics of Tycho's SNR well. In the case of the SNR expanding in the CSM the density and velocity structures are more complicated in comparison with the classical ISM case. The swept up mass of the CSM is about $2.0 \mathrm{M}_{\odot}$, in the ISM case the swept up mass is $\sim 1.4 \mathrm{M}_{\odot}$. The resulting thermal X-ray spectra differs drastically due to the different temperature and ionization timescale distributions throughout the shocked supernova ejecta. The fluxes of the emission lines and the locations of their centroids are defined by these parameters (for the detailed studies see e.g. Badenes et al. 2006). In the specific case of Tycho's SNR, the W7 explosion model in the wind bubble reproduces the observed spectra better (Figure 2, right column), while allowing for densities that give a consistent result for the kinematics. This result indicates that a non-homogeneous CSM may explain both the kinematics and the spectrum of Tycho SNR.

\section{Discussion}

While Kepler's SNR shows clear evidence for interaction with CSM, for Tycho's SNR the case is more subtle, as it manifests itself in the details of the X-ray spectrum. The reason may be that in Tycho the SNR blast-wave has already penetrated the shell.

Based on the kinematics and morphology of the Kepler's SNR and the chemical composition of its northern shell we argue that Kepler's SN had a symbiotic binary progenitor consisting of a CO WD and a $4-5 \mathrm{M}_{\odot}$ AGB donor star. For the case of Tycho's SNR, the presence of a dense non-homogeneous ambient medium around its progenitor seems be able to reconcile the differences that result from the studies of the kinematics and the X-ray spectra of the remnant. The specific structure and origin of this CSM needs further investigation.

Here we presented two cases which argue for stellar wind outflows around SNe Ia. There are several other studies suggesting similar outflows (e.g. Sternberg et al. 2011, Borkowski et al. 2006). However, direct radio, and X-ray observations of SNe Ia put rather stringent constraints on outflows from SNe Ia progenitors (e.g Mattila et al. 2005; Panagia et al. 2006; Immler et al. 2006). Reconciling these discrepancies is an important challenge for future SNe Ia studies. 

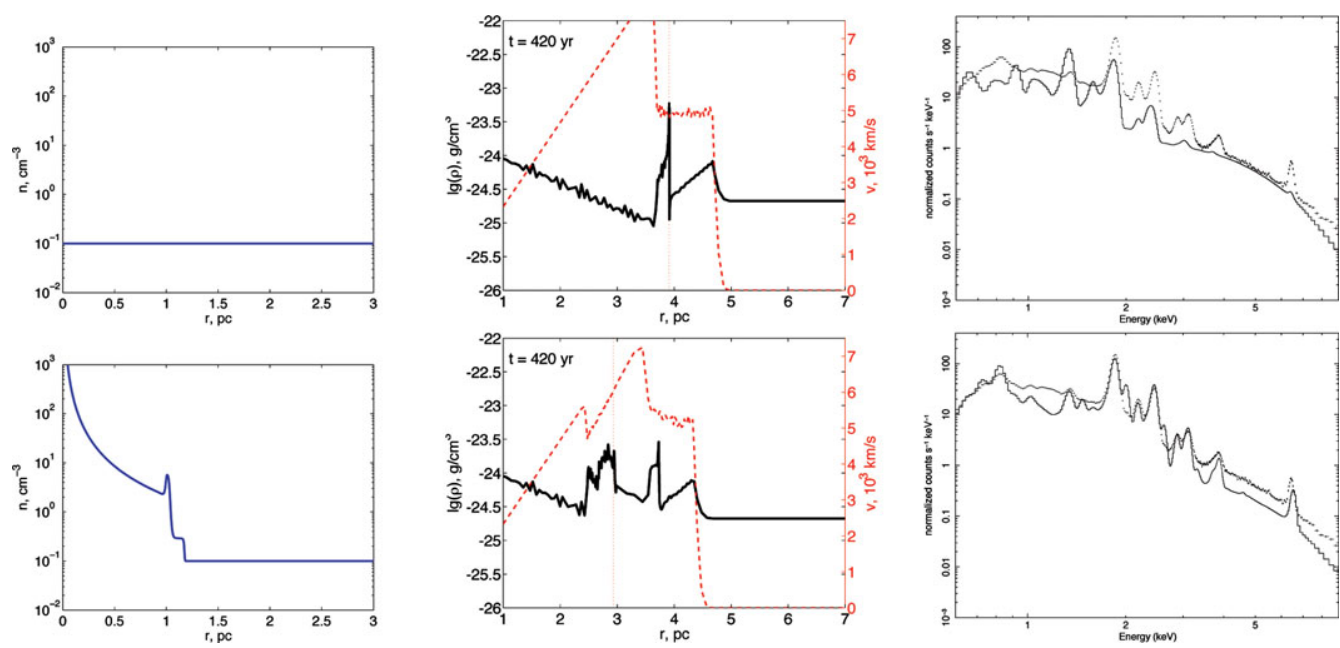

Figure 2. Left: the number density profiles of the ambient medium before the explosion. Middle: the properties of the SNR at the current age of Tycho's SNR (black solid line is the mass density, red dashed line is the velocity profile, vertical dotted line indicates the location of the contact discontinuity). Right: the X-ray spectrum for each model (solid lines) in comparison with the observed XMM-Newton spectra of Tycho's SNR (crosses). Note that X-ray synchrotron emission contributions (continuum) has not been included here. Top row: the case of the SNR evolution in a homogeneous ISM. Bottom row: the case of a SNR interacting with a wind bubble. The wind parameters are: mass loss rate $\dot{M}=10^{-5} \mathrm{M}_{\odot} \mathrm{yr}^{-1}$, wind velocity $u_{w}=10 \mathrm{~km} \mathrm{~s}^{-1}$ and the wind outflow phase lasts for 0.1 Myr. In both cases the ISM density is $\rho=2 \times 10^{-25} \mathrm{~g} \mathrm{~cm}^{-3}$ (Kosenko et al. in preparation).

\section{References}

Badenes, C., Borkowski, K. J., Hughes, J. P., Hwang, U., \& Bravo, E. 2006, ApJ, 645, 1373

Bandiera, R. 1987, ApJ, 319, 885

Bandiera, R. \& van den Bergh, S. 1991, ApJ, 374, 186

Blair, W. P., Long, K. S., \& Vancura, O. 1991, ApJ, 366, 484

Borkowski, K. J., Hendrick, S. P., \& Reynolds, S. P. 2006, ApJ, 652, 1259

Chiotellis, A., Schure, K. M., \& Vink, J. 2011, arXiv:1103.5487

Immler, S., Brown, P. J., Milne, P., et al. 2006, ApJ, 648, L119

Kaastra, J., Mewe, R., \& Nieuwenhuijzen, H. 1996, in UV and X-ray spectroscopy of astrophysical and laboratory plasmas (K. Yamashita and T. Watanabe), 411

Karakas, A. \& Lattanzio, J. C. 2007, PASA, 24, 103

Katsuda, S., Petre, R., Hughes, J. P., et al. 2010, ApJ, 709, 1387

Keppens, R., Nool, M., Tóth, G., \& Goedbloed, J. P. 2003, Computer Physics Communications, 153,317

Kosenko, D., Blinnikov, S. I., \& Vink, J. 2011, A $\mathcal{E} A$ 532, A114

Mattila, S., Lundqvist, P., Sollerman, J., et al. 2005, A\& $A$, 443, 649

Nomoto, K. 1984, ApJ, 277, 791

Panagia, N., Van Dyk, S. D., Weiler, K. W., et al. 2006, ApJ, 646, 369

Sollerman, J., Ghavamian, P., Lundqvist, P., \& Smith, R. C. 2003, A\& A, 407, 249

Sorokina, E., Blinnikov, S., Kosenko, D., \& Lundqvist, P. 2004, Astron. Lett., 30, 737

Sternberg, A., Gal-Yam, A., Simon, J. D., et al. 2011, Science, 333, 856

Vink, J. 2008, ApJ, 689, 231 